\author{
Rachel Margolis
}

A Partisan from Vilna 
JEWS OF POLAND

Series editor: Antony Polonsky

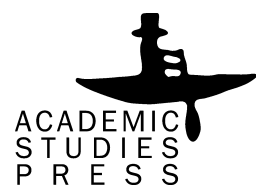




\section{Rachel Margolis}

\section{A Partisan from Vilna}

Translated by F. Jackson Piotrow

The translation of this book was made possible by the generosity of Erwin Greenberg.

Academic Studies Press

2010 
Library of Congress Cataloging-in-Publication Data

Margolis, Rahel.

[Nemnogo sveta vo mrake. English]

A partisan from Vilna / Rachel Margolis ; translated by F. Jackson Piotrow.

p. cm. -- (Jews of Poland)

Includes bibliographical references and index.

ISBN 978-1-934843-91-8 (hbk.) -- ISBN 978-1-934843-95-6 (pbk.) 1. Margolis, Rahel. 2. Women guerrillas--Lithuania--Biography. 3. Women guerrillas--Belarus--Biography. 4. World War, 1939-1945-Underground movements--Lithuania. 5. Fareynikte partizaner organizatsye (Vilnius, Lithuania)-Biography. 6. World War, 1939-1945--Underground movements--Belarus. 7. World War, 1939-1945-Personal narratives, Lithuanian. 8. World War, 1939-1945--Personal narratives, Jewish. 9. Jewish women--Lithuania--Vilnius--Biography. 10. Vilnius (Lithuania)--Biography. I. Title.

D802.L5M2813 2010

940.54'864793092--dc22

[B]

2010012946

Copyright $\odot 2010$ Academic Studies Press

All rights reserved

ISBN 978-1-934843-91-8 (cloth)

ISBN 978-1-934843-95-6 (paper)

Book design by Olga Grabovsky

On the cover: Remnants By Samuel Bak

Image Courtesy of Pucker Gallery www.puckergallery.com

Published by Academic Studies Press in 2010

28 Montfern Avenue

Brighton, MA 02135, USA

press@academicstudiespress.com

www.academicstudiespress.com 